\title{
UNC0638, a G9a inhibitor, suppresses epithelial-mesenchymal transition-mediated cellular migration and invasion in triple negative breast cancer
}

\author{
XIANG-RONG LIU ${ }^{1}$, LI-HUA ZHOU ${ }^{1}$, JIAN-XIN HU ${ }^{1}$, LI-MIN LIU ${ }^{1}$, HUI-PING WAN ${ }^{2}$ and XI-QUAN ZHANG ${ }^{2}$ \\ Departments of ${ }^{1}$ Pharmacy and ${ }^{2}$ Tumor, The People's Hospital of Jiangxi Province, Nanchang, Jiangxi 330006, P.R. China
}

Received July 4, 2017; Accepted October 5, 2017

DOI: $10.3892 / \mathrm{mmr} .2017 .8190$

\begin{abstract}
Triple-negative breast cancer (TNBC) is associated with an aggressive clinical history, high risk of recurrence and metastasis, and shorter patient survival due to lack of targeted therapy. In the present study, UNC0638, a chemical G9a inhibitor, was identified to suppress TNBC cell invasion and migration in vitro at a non-cytotoxic concentration. In addition, UNC0638 reduced the size and number of the tumorsphere and decreased anchor-independent colony formation in the two TNBC cell lines. Evaluation of the underlying mechanism revealed that the suppressive effect of UNC0638 is associated with modulation of epithelial-mesenchymal transition through enhancing E-cadherin promoter activities and restoring its expression. Thus, the current data indicates that UNC0638 may be developed as a chemotherapeutic agent to effectively treat metastatic cancers, including TNBC.
\end{abstract}

\section{Introduction}

Breast cancer is the most prevalent type of malignancy and the leading cause of cancer mortality among females worldwide (1). It's estimated that $\sim 1.7$ million new cases are diagnosed and $>500,000$ women succumb due to this disease annually (2). Depending on the expression of cellular markers, breast cancer is divided into three major subtypes: Estrogen receptor (ER)- or progesterone receptor (PR)-positive (luminal type), human epidermal grow th factor receptor 2 positive (HER2 type), and triple-negative breast cancer (TNBC) is defined by the lack of ER/PR and HER2 expression $(3,4)$. Therapeutic agents targeting ER/PR or HER 2 result in remarkable survival benefits. Tamoxifen for patients who express hormone receptors and trastuzumab for patients with overexpressed HER2 are typical examples of targeted therapy. However, TNBC

Correspondence to: Dr Li-Hua Zhou, Department of Pharmacy, The People's Hospital of Jiangxi Province, 92 Ai-Guo Road, Nanchang, Jiangxi 330006, P.R. China

E-mail: 540619077@qq.com

Key words: triple-negative breast cancer, G9a inhibitor, epithelialmesenchymal transition, E-cadherin, cancer stem-like cells has no standard treatment options, unlike other types of breast cancer (5). TNBC, accounting for $15-20 \%$ of all types of diagnosed breast cancer, is associated with an aggressive clinical history, high risk of recurrence and metastasis, and shorter patient survival (6). Due to the lack of targeted therapies, TNBC has a poor prognosis (7). Consequently, there is an urgent requirement to identify new targeted therapeutic agents to treat patients with TNBC.

Accumulating studies have demonstrated that the highly aggressive and metastatic characters of TNBC are associated with the aberrant activation of epithelial-mesenchymal transition (EMT), which is an essential process during embryonic development (8-10). During this EMT process, cells lose their epithelial phenotype, acquire fibroblast-like properties and exhibit reduced intercellular adhesion and increased motility, invasiveness and metastatic capability. Loss of E-cadherin expression and gain of mesenchymal markers, such as vimentin, are the main characteristics of EMT, which are also hallmarks of metastatic carcinoma (11-13). A previous study demonstrated that G9a is vital for the EMT process and the transcriptional repression of E-cadherin in human breast cancer and other types of epithelial carcinoma (14). Knockdown of G9a by short hairpin (sh)RNA restored E-cadherin expression and resulted in the inhibition of cell migration and invasion in vitro, and suppression of tumor growth and metastasis in vivo $(14,15)$. These findings encourage us to validate whether small molecular inhibition of G9a may inhibit the EMT-mediated invasion and migration of TNBC.

In the current study, UNC0638, a reported small molecular G9a inhibitor (16), suppressed invasion and migration in two TNBC cell lines, MDA-MB-231 and BT-549. Furthermore, UNC0638 inhibited the EMT process by repressing vimentin and transcriptional restoration of E-cadherin expression. These findings indicate that G9a inhibitors may be a promising therapeutic agent for TNBC treatment.

\section{Materials and methods}

Antibodies and reagents. UNC0638 was purchased from MedchemExpress LLC (Princeton, NJ, USA) and dissolved in dimethyl sulfoxide (DMSO). Dulbecco's modified Eagle's medium (DMEM) and fetal bovine serum (FBS) were purchased from Thermo Fisher Scientific, Inc. (Waltham, MA, USA). 3-(4,5-Dimethylthiazol-2-yl)-2,5-diphenyltetrazolium 
bromide (MTT) and low melt point soft agar were purchased from Sigma-Aldrich (Merck KGaA, Darmstadt, Germany). Invasion chambers and matrigel were purchased from BD Biosciences (Franklin Lakes, NJ, USA). Gentian violet was purchased from Beijing Solarbio Science \& Technology Co., Ltd. (Beijing, China). The Annexin V-fluorescein isothiocyanate/propidium iodide apoptosis detection kits (cat. no. 556570) were purchased from BD Biosciences. Cell lysis buffer (cat. No. P0013), a BCA protein assay kit (cat. no. P0011) and Primary Antibody Dilution Buffer (cat. no. P0023A) were purchased from Beyotime Institute of Biotechnology (Shanghai, China). Polyvinylidene fluoride (PVDF) membrane and chemiluminescent reagents were obtained from EMD Millipore (Billerica, MA, USA). Antibodies to E-cadherin (20874-1-AP), vimentin (10366-1-AP), Slug (12129-1-AP), Snail (13099-1-AP) and $\beta$-actin (60008-1-Ig) were purchased from Proteintech Group, Inc. (Chicago, IL, USA) and diluted at 1:1,000 with Primary Antibody Dilution Buffer (Beyotime, Beijing, China).

Cell culture and transfection. Human breast cancer cell lines, MDA-MB-231 and BT-549 were obtained from the American Type Culture Collection (Manassas, VA, USA) and cultured in DMEM medium supplemented with $10 \% \mathrm{FBS}$ and $1 \%$ penicillin/streptomycin in a $5 \% \mathrm{CO}_{2}$ atmosphere at $37^{\circ} \mathrm{C}$. The expression of E-cadherin was knocked down by stable transfection with lentivirus and selected with puromycin. The lentiviral vector, pLKO.1 puro shRNA E-cadherin was provided by Bob Weinberg (Addgene plasmid no. 18801) (17).

Cell viability assay. Cell viability was measured via MTT assay (cat. no. M2128 Sigma-Aldrich; Merck KGaA). A total of $5 \times 10^{3}$ cells per well were seeded into 96-well plates. After $24 \mathrm{~h}$ incubation at $37^{\circ} \mathrm{C}$, cells were treated with various concentrations of UNC0638 for $24 \mathrm{~h}$. At the end of drug treatment, $20 \mu \mathrm{l}$ per well MTT reagent $(5 \mathrm{mg} / \mathrm{ml}$ in phosphate-buffered saline; PBS) was added to each well and incubated at $37^{\circ} \mathrm{C}$ for $4 \mathrm{~h}$. Then the MTT-containing medium was replaced with $150 \mu \mathrm{l}$ DMSO per well and the plates were shaken for $10 \mathrm{~min}$ in the dark. Absorbance of each well was determined using a microplate reader at a wavelength of $490 \mathrm{~nm}$. All experiments were repeated three times.

Transwell assay. The cell migration and invasion assay were performed using the BD transwell system (cat. no. 353097; $\mathrm{BD}$ Biosciences) according to the manufacturer's protocols. Briefly, cells were treated with 0.5 and $1 \mu \mathrm{M}$ UNC0638 or diluent for $24 \mathrm{~h}$. Subsequently, $2 \times 10^{4}$ treated cells in $200 \mu \mathrm{l}$ serum-free medium were seeded in the upper chambers of the transwell inserts and culture medium with $10 \%$ FBS was added to the lower chambers. For the invasion assay, inserts were pre-coated with Matrigel. After $24 \mathrm{~h}$ incubation at $37^{\circ} \mathrm{C}$, cells that had migrated to the reverse side of the inserts were fixed with methanol and stained with Gentian violet at room temperature for $30 \mathrm{~min}$. Cells in randomly selected fields were counted under an inverted microscope (Olympus CKX31; Olympus Corporation, Tokyo, Japan).

Wound-healing assay. Cells were seeded in 6-well plates and grown into a confluent monolayer in complete medium.
An artificial wound across the cell culture monolayer was formed by scratching with a $10-\mu 1$ pipette tip. The wounded monolayers were then washed twice with PBS to remove non-adherent cells, and serum-free medium with or without UNC0638 was added to the 6-well plate. Images of each wounded monolayer were obtained at 0 and $24 \mathrm{~h}$ using a phase-contrast microscope.

Western blotting. Following treatment with UNC0638 or diluent, cells were harvested and lysed with cell lysis buffer. The concentration of total protein was determined using the BCA protein assay kit. Equal quantities (30 $\mu \mathrm{g}$ per load) of protein were size-fractionated by $10 \%$ SDS-polyacrylamide gel electrophoresis and transferred onto PVDF membranes $(90 \mathrm{~V}$ for $2 \mathrm{~h}$ ). After blocking with $10 \%$ non-fat milk, the membrane was reacted with primary antibodies at $4^{\circ} \mathrm{C}$ overnight, followed by incubation with horseradish peroxidase-conjugated secondary antibodies at room temperature for $2 \mathrm{~h}$. The protein bands were detected using an enhanced chemiluminescence detection system (cat. no. WBKLS0500; Merck KGaA) according to the manufacturer's protocols.

Tumorsphere culture. Cells $\left(10^{5}\right.$ per well) were plated in ultra-low attachment 6-well plate (Corning Incorporated, Corning, NY, USA) in serum-free DMEM-F12 medium supplemented with $20 \mathrm{ng} / \mathrm{ml}$ basic fibroblast growth factor, $20 \mathrm{ng} / \mathrm{ml}$ epidermal growth factor, $5 \mu \mathrm{g} / \mathrm{ml}$ insulin and $0.4 \%$ bovine serum albumin. Following incubation at $37^{\circ} \mathrm{C}$ for $48 \mathrm{~h}$, UNC0638 or diluent was added to the medium and the suspension cultures continued for 1-2 weeks until tumorspheres formed. Tumorspheres with diameter $>50 \mu \mathrm{m}$ were counted. The colony numbers were obtained from five random views using a microscope. Experiments were repeated three times with duplication in each experiment.

Soft-agar and tumorigenesis assays. The 6-well plate was pre-coated with $1 \mathrm{ml}$ per well $2 \%$ low-melting agarose. Following treatment with 0.5 and $1 \mu \mathrm{M} \mathrm{UNC} 0638$ or diluent for $24 \mathrm{~h}$, cells were detached, counted and resuspended in $0.5 \mathrm{ml}$ $1 \%$ low-melting-point agarose mixed with $0.5 \mathrm{ml} 2 \mathrm{X}$ complete culture medium. The cell suspensions were then layered on top of $2 \%$ low-melting agarose and incubated at $37^{\circ} \mathrm{C}$ in a humidified atmosphere of $5 \% \mathrm{CO}_{2}$ for 2 weeks. Colonies containing at least 50 cells were counted. All experiments were repeated three times.

Luciferase reporter assay. The E-cadherin promoter sequences (from-108 to 125 of the human $E$-cadherin gene) were synthesized by Genewiz, Inc. (Suzhou, China) and cloned into pGL3-basic vector (18). For the luciferase assay, $2 \times 10^{5}$ cells per well were seeded in a 6 -well plate. Following incubation at $37^{\circ} \mathrm{C}$ for $24 \mathrm{~h}$, cells were co-transfected with E-cadherin promoter-luciferase plasmid together with pRL-CMV (Renilla luciferase) using Lipofectamine ${ }^{\circledR} 2000$ transfection agents (cat. no. 11668-019; Invitrogen; Thermo Fisher Scientific, Inc.). After $6 \mathrm{~h}$, the medium was replaced with fresh medium containing UNC0638 or diluent and cultured for another $24 \mathrm{~h}$. luciferase activity was measured using the Dual-Luciferase Assay kit (cat. no. E1910; Promega Corporation, Madison, WI, USA) according to the manufacturer's protocol. The firefly 

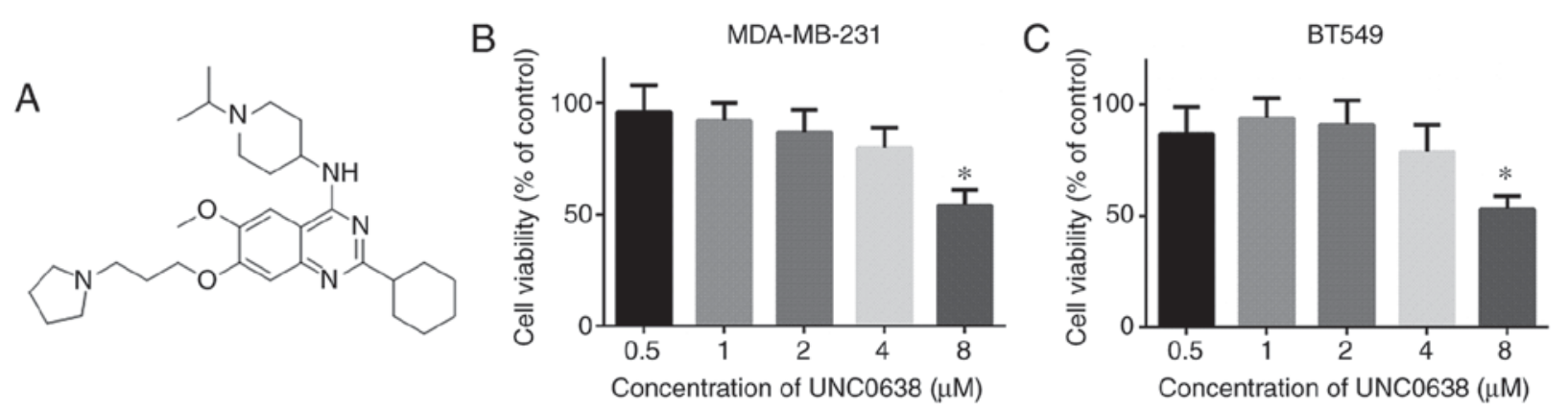

Figure 1. Effects of UNC0638 on the cell viability. (A) Chemical structure of UNC0638. The cell viability of (B) MDA-MB-231 and (C) BT-549 was determined by 3-(4,5-dimethylthiazol-2-yl)-2,5-diphenyltetrazolium bromide assay following treatment with various concentrations (0-8 $\mu \mathrm{M})$ of UNC0638 for $24 \mathrm{~h}$. The results are presented as means \pm standard deviation of three independent experiments. ${ }^{\text {" }} \mathrm{P}<0.05 \mathrm{vs}$. control (DMSO treated group).

luciferase activity value was normalized to the Renilla activity value. All procedures were performed in triplicate.

Statistical analysis. All experiments were repeated three times and statistical analysis was performed using SPSS software (version 19; IBM Corp., Armonk, NY, USA). Data were analyzed using Student's t-tests and $\mathrm{P}<0.05$ was considered to indicate a statistically significant difference.

\section{Results}

UNC0638 suppresses the migration and invasion of TNBC cells. UNC0638 was identified as a synthetic small molecular compound with potent inhibitory activities against G9a (Fig. 1A). In the present study, the cytotoxicity of UNC0638 was initially determined in two TNBC cell lines, MDA-MB-231 and BT-549. The two cell lines were treated with UNC0638 at various concentrations (ranging from $0.5-8 \mu \mathrm{M}$ ) for $24 \mathrm{~h}$ followed by MTT assay. In comparison with that of the solvent control (DMSO), the cellular viabilities of the two TNBC cell lines were not significantly affected by treating with UNC0638 at a concentration between 0.5 and $4 \mu \mathrm{M}$ (Fig. 1B and C). Therefore, non-cytotoxic concentrations of UNC0638 $(<4 \mu \mathrm{M})$ were applied in all subsequent experiments.

Metastasis, which is the critical reason for treatment failure and poor prognosis in patients with TNBC, involves migrations and invasive abilities of cancer cells to pass through the basement membrane and to invade surrounding tissues $(19,20)$. Therefore, a transwell assay was performed to examine the effect of UNC0638 on the migratory and invasive ability of TNBC cells. For detection of cell invasive ability, a chamber membrane was pre-coated with Matrigel to stimulate the basement membrane. As demonstrated in Fig. 2A-D, the number of cells migrating to the lower chamber and invading the Matrigel membrane were significantly reduced by UNC0638 in a dose-dependent manner.

The inhibitory effect of UNC0638 on migration of MDA-MB-231 and BT-549 cells was evaluated by wound-healing assay. After a single layer of cells was scratched to generate the wounds, the two TNBC cell lines were treated with 0.5 and $1 \mu \mathrm{M}$ UNC0638 for $24 \mathrm{~h}$. As demonstrated in Fig. 2E and F, UNC0638 treatment significantly decreased the ability of TNBC cells to migrate and recover the wounded area. The quantitative data presented in Fig. $2 \mathrm{H}$ indicated that TNBC inhibited the migration of TNBC cells in a dose-dependent manner. These results indicate that UNC0638 is effective in preventing TNBC cell migration and invasion.

G9a promotes EMT activation of TNBC cells by directly repressing the expression of E-cadherin $(14,21,22)$. Therefore, in the following experiment, the effect of UNC0638 on the expression of various EMT markers was determined in the two TNBC cell lines. As shown in Fig. 2I, the expression levels of E-cadherin were dose-dependently upregulated by UNC0638 treatment in the two cell lines, whereas the expression levels of vimentin were downregulated. In addition, UNC0638 treatment decreased the expression level of Slug and Snail1 in the two cell lines, which are key transcription factors that strongly promote the EMT process.

UNC0638 inhibits cancer stem-like cell (CSC) properties of $T N B C$ cells. The invasive and metastatic features of TNBC cells are closely linked to the EMT activation and CSC properties. Acquisition of CSC activities block cellular differentiation and promote cancer cells to convert from their differentiated epithelial state to a mesenchymal phenotype, which provides a distinct advantage in tumor progression and metastasis $(23,24)$. As undifferentiated CSCs were hypothesized to generate tumorspheres in non-adhesive and serum-free culture conditions, tumorsphere assays are useful for enriched CSCs and investigating their behavior $(25,26)$. In the present study, MDA-MB-231 and BT-549 cells were cultured in serum-free suspension conditions in the presence or absence of UNC0638 for a week, and the number and size of tumorspheres were monitored under phase contrast microscopy. As presented in Fig. $3 \mathrm{~A}$ and $\mathrm{B}$, the size and numbers of tumorspheres were significantly decreased by UNC0638 indicating its inhibitory effect on tumorsphere generation of TNBC cells.

A critical feature of CSCs is the self-renewal capacity, which enables a single cell to form a colony under anchorage independent conditions $(27,28)$. In the current study, a soft agar colony assay was used to determine the effect of UNC0638 on the CSC self-renewal capacity. As summarized in Fig. 3C, the numbers of colonies formed by UNC0638-treated cells are dose-dependently decreased, when compared with the cells treated with diluent (DMSO). In addition, the size of the colonies formed in UNC0638-treated groups were markedly smaller than that in the untreated groups (data not shown). Together, these results demonstrate that UNC0638 exhibited potent inhibitory activities on tumorigenicity and self-renewal of breast CSCs. 

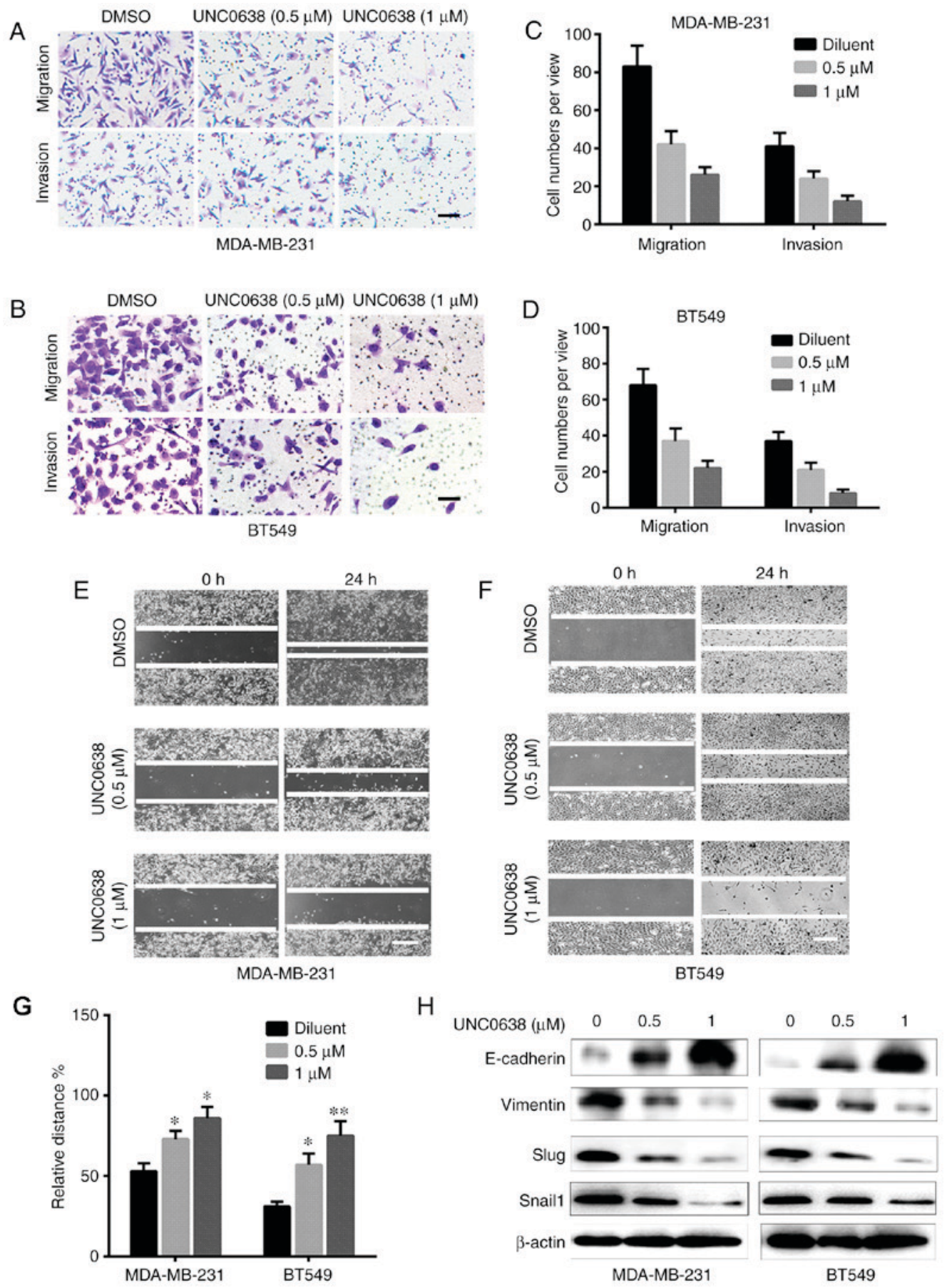

Figure 2. UNC0638 suppressed triple negative breast cancer cell migration and invasion by regulating EMT-associated proteins. Effects of UNC0638 on the migration and invasion of (A) MDA-MB-231 and (B) BT-549 cells determined by transwell assay. For the cell invasion assay, the upper chambers of transwell inserts were pre-coated with Matrigel. Quantitative analysis of migratory and invaded (C) MDA-MB-231 and (D) BT-549 cells. Cells were stained with Gentian violet. Scale bars $=20 \mu \mathrm{m}$. Detection of migration of (E) MDA-MB-231 and (F) BT-549 cells by wound healing assay. Scale bars=100 $\mu \mathrm{m}$. (G) Quantification of wound healing. (H) Western blots of EMT-associated proteins. All experiments were repeated three times. "P<0.05 and ${ }^{* *} \mathrm{P}<0.01$ vs. control. EMT, epithelial-mesenchymal transition; DMSO, dimethyl sulfoxide.

UNC0638 suppresses the G9a-induced EMT process that is dependent on restoring E-cadherin. In addition, E-cadherin was silenced in the two TNBC cell lines to determine whether restoring E-cadherin is essential to UNC0638 inhibitory activities against EMT-mediated invasion and migration of TNBC cells. As presented in Fig. 4A, shRNA stable transfection efficiently attenuates the UNC0638 induced upregulation of E-cadherin. Furthermore, E-cadherin knockdown restores the repressive effect of UNC0638 on cellular migration and invasion (Fig. 4B and C). In addition, as studies have revealed that G9a, combined with other transcription factors, directly binds to the promoter of E-cadherin and represses its mRNA transcription, the effect of the G9a inhibitor on mRNA expression levels and promoter activity of E-cadherin was examined in the MDA-MB-231 cell line. As presented in Fig. 4D and E, UNC0638 dose-dependently increased the mRNA expression levels and promoter activity of E-cadherin. Thus, these results indicate that UNC0638 
A

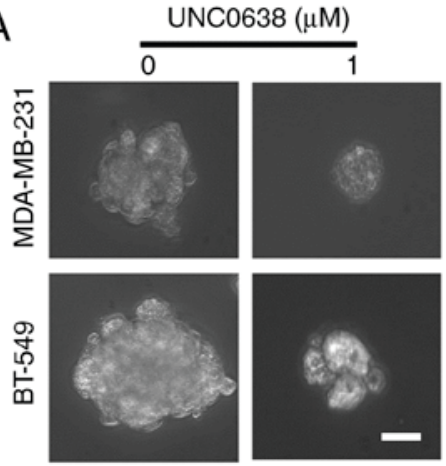

B

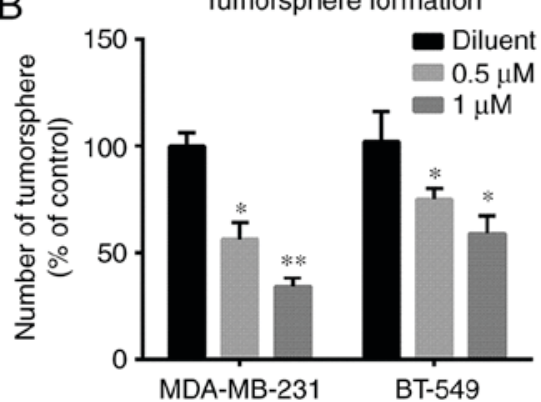

$\mathrm{C}$

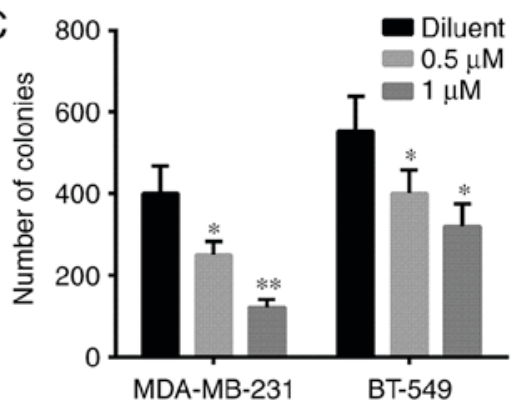

Figure 3. UNC0638 inhibits cancer stem-like cells properties of triple negative breast cancer cells. (A) Representative images of tumorsphere formed by cells treated with or without UNC0638. Scale bars $=20 \mu \mathrm{m}$ Numbers of (B) tumorsphere and (C) anchorage-independent colony formation. The experiments were repeated three times. ${ }^{*} \mathrm{P}<0.05$ and ${ }^{* *} \mathrm{P}<0.01$ vs. control.
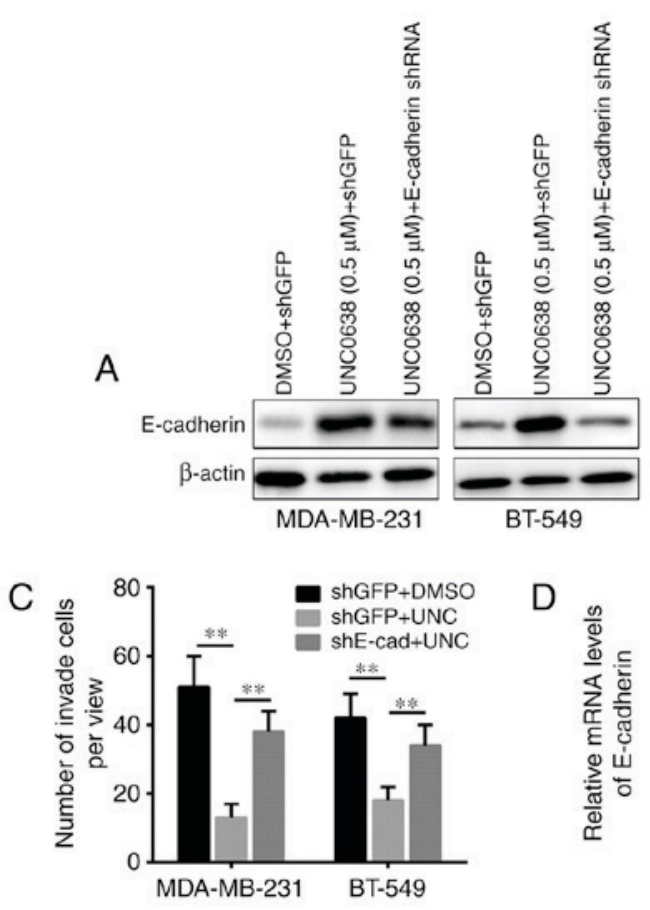

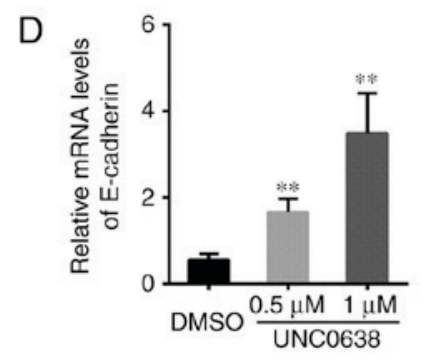

Figure 4. UNC0638 suppresses cell invasion by restoring E-cadherin expression levels. (A) Silencing of E-cadherin by lentivirus-dependent shRNA. (B) Knockdown of E-cadherin significantly attenuates UNC0638-induced inhibition of cell invasion. Cells were stained with Gentian violet. Scale bars $=20 \mu \mathrm{m}$. (C) Quantitative analysis of invaded cells. (D) Effect of UNC0638 on the mRNA expression level of E-cadherin in MDA-MB-231 cells. (E) Effect of UNC0638 on the E-cadherin promotor activity in MDA-MB-231 cells. The experiments were repeated three times. ${ }^{* *} \mathrm{P}<0.01$ vs. control. shRNA, short hairpin RNA; DMSO, dimethyl sulfoxide; GFP, green fluorescence protein.

exhibited its inhibitory activities by restoring E-cadherin transcription.

\section{Discussion}

The EMT, which is considered to be the most important step in the metastatic process of epithelial tumors, is associated with the decreasing or complete loss of E-cadherin expression. Various transcription repressors, such as Snail, Slug, Twist and zinc finger E-box binding homeobox 1, have been implicated in the transcriptional repression of E-cadherin and the induction of EMT $(11,29)$. Studies have revealed that Snail interacted with G9a, and recruited G9a and DNA methyltransferases to the E-cadherin promoter for DNA methylation, leading to transcriptional silencing of E-cadherin in breast cancer cells. Knockdown of G9a by shRNA reversed EMT markers and inhibited cellular migration and invasion in breast cancer, head and neck squamous cell carcinoma $(14,15)$. In addition, snail-G9a complex was identified to repress the expression of fructose-1,6-biphosphatase and enhance cellular invasiveness in breast cancer cells (18). In the present study, a chemical inhibitor of G9a, UNC0638, was demonstrated to suppress TNBC cell migration and invasion at a non-cytotoxic dose, and reversed the expression levels of various EMT markers, including E-cadherin and vimentin. Notably, it was demonstrated that UNC0638 upregulated E-cadherin by enhancing its promoter activity. The present study demonstrated that the anti-migration and anti-invasion activities of UNC0638 were 
dependent upon restoring the expression level of E-cadherin. Similar to the present results, Liu et al (15) indicated that inhibition of G9a by another chemical compound, BIX suppressed cellular migration with upregulation of E-cadherin in head and neck squamous cell carcinoma. Thus, a Snail-G9a axis may be developed as an effective therapeutic target for treating cancer metastasis and recurrence.

The current study identified that UNC0638 reduced the size and number of tumorspheres under non-adhesive conditions and suppressed anchorage-independent colony formation, which represents the inhibition of CSC properties. CSCs are hypothesized to contribute to tumor metastasis and therapeutic resistance, as CSCs display EMT characteristics, such as loss of the adhesion protein, E-cadherin and acquisition of high motility (29). Thus, UNC0638 may inhibit CSC properties via conversion of Snail-G9a induced EMT in TNBC cells.

In conclusion, UNC0638 blocks CSC properties and the EMT program by targeting the Snail-G9a axis and restoring E-cadherin in TNBC cells. As a result, the cellular invasiveness and motility were decreased by TNBC treatment in MDA-MB-231 and BT-549 cells. Thus, this compound may be developed as a potential therapeutic agent to treat metastatic TNBC subsequent to further in vivo and pharmacokinetic validation.

\section{References}

1. Siegel R, Ma J, Zou Z and Jemal A: Cancer statistics, 2014. CA Cancer J Clin 64: 9-29, 2014

2. Ferlay J, Soerjomataram I, Dikshit R, Eser S, Mathers C, Rebelo M, Parkin DM, Forman D and Bray F: Cancer incidence and mortality worldwide: Sources, methods and major patterns in GLOBOCAN 2012. Int J Cancer 136: E359-386, 2015.

3. de Ruijter TC, Veeck J, de Hoon JP, van Engeland M and Tjan-Heijnen VC: Characteristics of triple-negative breast cancer. J Cancer Res Clin Oncol 137: 183-192, 2011.

4. Tomao F, Papa A, Zaccarelli E, Rossi L, Caruso D, Minozzi M, Vici P, Frati L and Tomao S: Triple-negative breast cancer: New perspectives for targeted therapies. Onco Targets Ther 8: 177-193, 2015.

5. Rota LM and Wood TL: Crosstalk of the insulin-like growth factor receptor with the Wnt signaling pathway in breast cancer. Front Endocrinol (Lausanne) 6: 92, 2015.

6. Voduc KD, Cheang MC, Tyldesley S, Gelmon K, Nielsen TO and Kennecke H: Breast cancer subtypes and the risk of local and regional relapse. J Clin Oncol 28: 1684-1691, 2010.

7. Bandyopadhyay S and Ali-Fehmi R: Breast carcinoma: Molecular profiling and updates. Clin Lab Med 33: 891-909, 2013.

8. Sarrió D, Rodriguez-Pinilla SM, Hardisson D, Cano A, Moreno-Bueno G and Palacios J: Epithelial-mesenchymal transition in breast cancer relates to the basal-like phenotype. Cancer Res 68: 989-997, 2008.

9. Polyak K: Heterogeneity in breast cancer. J Clin Invest 121 : 3786-3788, 2011.

10. Jeong H, Ryu YJ, An J,Lee Y and Kim A: Epithelial-mesenchymal transition in breast cancer correlates with high histological grade and triple-negative phenotype. Histopathology 60: E87-95, 2012.

11. Thiery JP: Epithelial-mesenchymal transitions in tumour progression. Nat Rev Cancer 2: 442-454, 2002.
12. Wang Y, Shi J, Chai K, Ying X and Zhou BP: The Role of Snail in EMT and Tumorigenesis. Curr Cancer Drug Targets 13: 963-972, 2013.

13. Lamouille S, Xu J and Derynck R: Molecular mechanisms of epithelial-mesenchymal transition. Nat Rev Mol Cell Biol 15: 178-196, 2014.

14. Dong C, Wu Y, Yao J, Wang Y, Yu Y, Rychahou PG, Evers BM and Zhou BP: G9a interacts with Snail and is critical for Snail-mediated E-cadherin repression in human breast cancer. J Clin Invest 122: 1469-1486, 2012.

15. Liu S, Ye D, Guo W, Yu W, He Y, Hu J, Wang Y, Zhang L, Liao Y, Song H, et al: G9a is essential for EMT-mediated metastasis and maintenance of cancer stem cell-like characters in head and neck squamous cell carcinoma. Oncotarget 6: 6887-6901, 2015.

16. Vedadi M, Barsyte-Lovejoy D, Liu F, Rival-Gervier S, Allali-Hassani A, Labrie V, Wigle TJ, Dimaggio PA, Wasney GA, Siarheyeva A, et al: A chemical probe selectively inhibits G9a and GLP methyltransferase activity in cells. Nat Chem Biol 7: 566-574, 2011.

17. Onder TT, Gupta PB, Mani SA, Yang J, Lander ES and Weinberg RA: Loss of E-cadherin promotes metastasis via multiple downstream transcriptional pathways. Cancer Res 68: 3645-3654, 2008.

18. Dong C, Yuan T, Wu Y, Wang Y, Fan TW, Miriyala S, Lin Y, Yao J, Shi J, Kang T, et al: Loss of FBP1 by Snail-mediated repression provides metabolic advantages in basal-like breast cancer. Cancer Cell 23: 316-331, 2013.

19. Rakha EA and Chan S: Metastatic triple-negative breast cancer. Clin Oncol (R Coll Radiol) 23: 587-600, 2011.

20. Kumar P and Aggarwal R: An overview of triple-negative breast cancer. Arch Gynecol Obstet 293: 247-269, 2016.

21. Lin Y, Dong C and Zhou BP: Epigenetic regulation of EMT: The Snail story. Curr Pharm Des 20: 1698-1705, 2014.

22. Hsiao SM, Chen MW, Chen CA, Chien MH, Hua KT, Hsiao M, Kuo ML and Wei LH: The H3K9 Methyltransferase G9a Represses E-cadherin and is associated with myometrial invasion in endometrial cancer. Ann Surg Oncol 22 (Suppl 3): S1556-S1565, 2015.

23. Luo M, Brooks M and Wicha MS: Epithelial-mesenchymal plasticity of breast cancer stem cells: Implications for metastasis and therapeutic resistance. Curr Pharm Des 21: 1301-1310, 2015.

24. Wu Y, Sarkissyan M and Vadgama JV: Epithelial-mesenchymal transition and breast cancer. J Clin Med 5: pii: E13, 2016.

25. Fillmore CM and Kuperwasser C: Human breast cancer cell lines contain stem-like cells that self-renew, give rise to phenotypically diverse progeny and survive chemotherapy. Breast Cancer Res 10: R25, 2008.

26. Singh JK, Farnie G, Bundred NJ, Simões BM, Shergill A, Landberg G, Howell SJ and Clarke RB: Targeting CXCR1/2 significantly reduces breast cancer stem cell activity and increases the efficacy of inhibiting HER2 via HER2-dependent and -independent mechanisms. Clin Cancer Res 19: 643-656, 2013.

27. Hwang-Verslues WW, Kuo WH, Chang PH, Pan CC, Wang HH, Tsai ST, Jeng YM, Shew JY, Kung JT, Chen CH, et al: Multiple lineages of human breast cancer stem/progenitor cells identified by profiling with stem cell markers. PLoS One 4: e8377, 2009.

28. Horibata S, Vo TV, Subramanian V, Thompson PR and Coonrod SA: Utilization of the soft agar colony formation assay to identify inhibitors of tumorigenicity in breast cancer cells. $\mathrm{J}$ Vis Exp e52727, 2015.

29. Thiery JP, Acloque H, Huang RY and Nieto MA: Epithelial-mesenchymal transitions in development and disease. Cell 139: 871-890, 2009.

This work is licensed under a Creative Commons Attribution-NonCommercial-NoDerivatives 4.0 International (CC BY-NC-ND 4.0) License. 